Ann. Zootech., I974, $23(4), 445^{-457}$.

\title{
INFLUENCE DE LA FRÉQUENCE DES TRAITES ET DES TÉTÉES SUR LA PRODUCTION LAITIÈRE DES BREBIS PREALPES DU SUD
}

\author{
J. LABUSSIÈRE, J.-F. COMBAUD et P. PETREQUIN \\ avec la collaboration technique de P. ChEsne \\ Laboratoire de Recherches sur la Physiologie et la Technologie de la Traite, \\ Centre de Recherches de Rennes, I. N.R.A., \\ 35042 Rennes Cedex

\section{RÉSUMÉ}

Les effets du nombre de tétées et de traites mécaniques sont étudiés séparément à l'aide de 2 carrés latins $6 \times 6$ comprenant chacun :

- 6 traitements : I, 2, 3, 4, 5 et 7 traites ou tétées par 24 heures( les horaires en sont précisés au tableau I.)

- 6 périodes de ro jours à l'exception de la première d'entre elle qui dure I $_{5}$ jours et qui n'étant pas influencée par un traitement précédent, constitue à elle seule une comparaison expérimentale très intéressante.

- 6 groupes de brebis Préalpes $d u$ Sud dont la composition est fixée au tableau 2 (2oo animaux en tout).

Les résultats de cet essai sont rapportés aux figures 2 et 3 ainsi qu'aux tableaux 3 et 4 . Leur examen indique qu'à fréquence égale de traite et de tétée l'efficacité d'un agneau "étranger " n'est pas supérieure à celle de la machine à traire lorsque le travail de celle-ci est complété par une intervention manuelle du trayeur.

Par rapport au traitement 2 choisi arbitrairement comme témoin, la pratique d'une seule traite ou d'une seule tétée par jour entraîne une réduction importante de la production (respectivement 55,7 p. Ioo et $5 \mathrm{I}, 3 \mathrm{p}$. roo lorsque la comparaison porte sur la I re période et $29,7 \mathrm{p}$. Ioo et $34,7 \mathrm{p}$. Ioo quand on prend en considération les valeurs enregistrées sur l'ensemble du carré latin).

Inversement le passage à des fréquences supérieures entraîne des gains relativement limités (I 2 à I 5 p. Ioo entre le traitement 2 /jour et $3 /$ jour).

La différence de production obtenue entre 7 traites et 2 tétées permet d'expliquer pour une grande part la perte de production laitière habituellement constatée au moment du sevrage.

\section{I. - INTRODUCTION}

Peu de travaux ont été consacrés à l'influence du nombre de tétées journalières sur la production laitière de la Brebis. Ils conduisent généralement à des interprétations contradictoires. C'est ainsi que pour connaître d'une façon rigoureuse la 
quantité de lait produite pendant la phase d'allaitement on admet généralement qu'il faut respecter un intervalle de 2 à 4 heures entre chaque contrôle de tétée.

Bien que pour McCANCE (I959) la synthèse du lait est déjà réduite à partir de ces délais il est probable que ce choix est surtout imposé par le souci de faire consommer à l'agneau le maximum du lait présent dans la mamelle (Munro et Inkson, I957). Si cette condition n'était pas remplie on risquerait en effet, de sous estimer l'activité ecrétoire qui n'est semble-t-il réellement freinée qu'au-delà de la $\mathrm{I} 2^{\mathrm{e}}$ heure (MoRAG, I969) et peut-être même seulement après la I $6^{\mathbf{e}}$ heure (SEMJAN, I g62).

Les quelques études sur la fréquence journalière des traites, aboutissent également à des résultats divergents qui peuvent non seulement s'expliquer par certaines imperfections expérimentales (périodes trop courtes, taille insuffisante du troupeau) mais aussi par la multiplicité des races utilisées.

En effet, si Bagdasarov (I960) et Morag (r968) admettent tous les deux une perte d'environ $\mathbf{I} 8 \mathrm{p}$. Ioo lorsqu'on compare une traite à deux traites, on constate par contre de grandes différences lors du passage de 2 à 3 traites puisque celui-ci n'aurait aucune incidence sur la production laitière pour MoRAG (Ig66 et I968) alors qu'il entraîne un rendement supplémentaire respectivement de 4,06 p. Ioo, 22,6 p. Ioo et 57,8 p. Ioo pour Srmuan (I96I), GAAI. (I957) et SVEC (I966).

Enfin Grigorov et Shalichev (I962) estiment que le gain est encore très appréciable entre la $3^{\mathrm{e}}$ et la $4^{\mathrm{e}}$ traite (environ $\mathrm{I} 4 \mathrm{p}$. IOO) mais nous n'avons pu trouver d'autres données susceptibles d'infirmer ou de confirmer leurs résultats.

Compte tenu de toutes les remarques précédentes et comme à notre connaissance il n'existe jusqu'à maintenant aucun essai sur des fréquences supérieures il nous a paru justifié d'étudier l'influence de six rythmes journaliers de traites et de tétées sur la production laitière des brebis. Nous avons choisi des animaux Préalpes $d u$ Sud car un certain nombre d'arguments expérimentaux permettent de supposer que leur secrétion laitière est fortement freinée lorsqu'on accroît l'intervalle qui sépare 2 traites ou 2 tétées.

C'est ainsi par exemple que dans cette race:

$\mathrm{I}^{\circ}$ les perturbations provoquées par la suppression de la traite du dimanche soir (LABUSSIÈre, CombaUd et PETREQUIN, I974) sont beaucoup plus importantes que celles ressenties chez la Brebis Sarde (CAsu et LABussière, I972);

$2^{\circ}$ la perte de production laitière constatée au moment du sevrage est toujours très élevée (environ 30 p. IOO : LABUSSIÈRE et PETREQUIN, I969) et on ne peut manquer de rappeler à ce sujet que la traite mécanique n'est effectuée que 2 fois par jour alors que le nombre de tétées quotidiennes peut varier entre 6 et 20 selon l'âge et le poids de (ou des) agneaux (Ricordeau, Boccard et DENAMUr, I960). L'objet de cette note est de rapporter les résultats obtenus en I 970 sur le troupeau expérimental du domaine I. N. R. A. de Brotuessy (78).

\section{II. - MATÉRIEL, ET MÉTHODES}

\section{A. - Présentation générale de l'essai et entrée des animaux en expérience}

Les effets de fréquences de tétée et de traite sont étudiés séparément à l'aide de 2 carrés latins $6 \times 6$ comprenant chacun 6 traitements, 6 périodes et 6 groupes de brebis.

Pour des raisons d'organisation du travail les animaux n'ont pas tous agnelé simultanément 
ce qui explique la légère torsion observée sur la représentation graphique des carrés latins qui sont présentés à la figure $\mathbf{r}$.

\begin{tabular}{|c|c|c|c|c|c|c|}
\hline \multirow{2}{*}{$\begin{array}{l}\text { DESIGNATION } \\
\text { DES GROUPES }\end{array}$} & \multicolumn{6}{|c|}{ TETEES } \\
\hline & $A$ & $B$ & $C$ & $D$ & $E$ & $\bar{F}$ \\
\hline $\begin{array}{l}\text { ECART ENTRE LA } \\
\text { MISE BAS et L'ES } \delta \text { SA }\end{array}$ & 4,6 & 4,3 & 7,8 & 7,9 & 7,7 & 8,1 \\
\hline
\end{tabular}

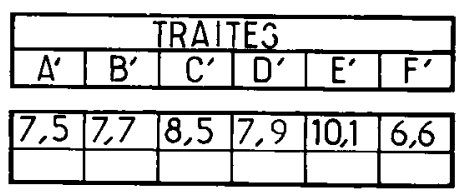

\section{JANVIER}
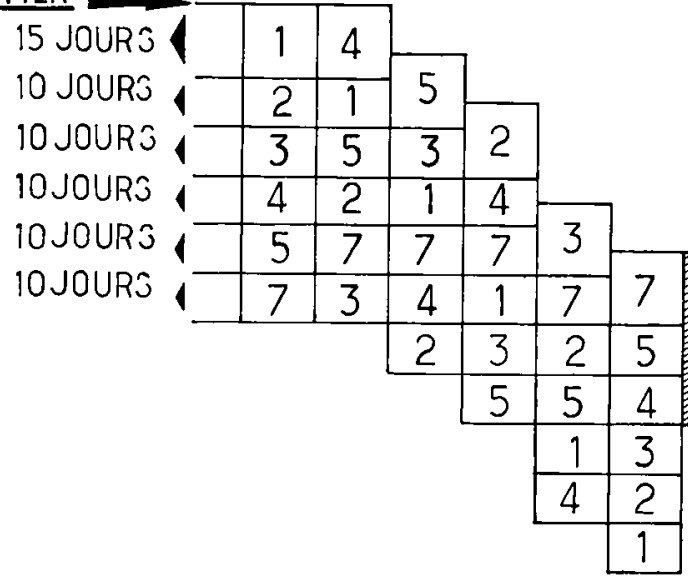

16 JUIN

FIg. I. - Représentation graphique des deux plans expérimentaux en carré latin

(L'écart entre la mise bas et l'essai est exprimé en jours)

Malgré tout on remarquera qu'il existe toujours un écart d'environ une semaine entre la mise bas et le début de l'expérience proprement dite au cours duquel les brebis sont maintenues en présence de leur jeune dans des cages spécialement aménagées à cet effet.

\section{B. - Traitements}

Le premier carré latin permet de comparer $1,2,3,4,5$ et 7 tétées par jour. Le second, très semblable au précédent, intéresse le rythme de traite ( $\mathrm{I}, 2,3,4,5$ et 7 traites/jour).

Les horaires de ces 2 types d'intervention sont précisés au tableau $\mathbf{I}$.

\section{Contrôle de la quantité de lait tété.}

Après la semaine d' "allaitement protégé " qui suit la mise bas les agneaux sont définitivement séparés de leur mère et celles-ci sont donc tétées pendant toute la durée de l'essai par des "étrangers" dont l'âge correspond approximativement à leur stade de lactation. On comprend en effet que pour ne pas fausser l'expérience il était indispensable d'organiser un plan d'alimentation des jeunes qui régularise le nombre de leurs repas journaliers $\left(^{\mathbf{1}}\right)$.

(1) Ceci n'aurait évidemment pas été possible avec les agneaux maternels auxquels on aurait nécessaisrement imposé un nombre de tétées semblable à celui de leur mère. 
Les tentatives de rejet des agneaux sont surtout fréquentes pendant les premiers jours et nécessitent alors la présence d'un ouvrier pour maintenir la brebis. Après une semaine environ, les "refus" sont de plus en plus rares et le rôle du berger consiste essentiellement alors :

- à peser l'agneau avant et après la tétée (précision voisine de $5 \mathrm{~g}$ ) et à surveiller ses déjections pour corriger les chiffres précédents (environ $30 \mathrm{~g}$ pour l'urine et $10 \mathrm{~g}$ pour les fèces).

- à contrôler manuellement si la mamelle a été complètement vidée et éventuellement à rajouter un deuxième (et parfois un troisième) agneau pour terminer l'évacuation de la glande.

\section{TABLEAU I}

Horaive des traites et tétées

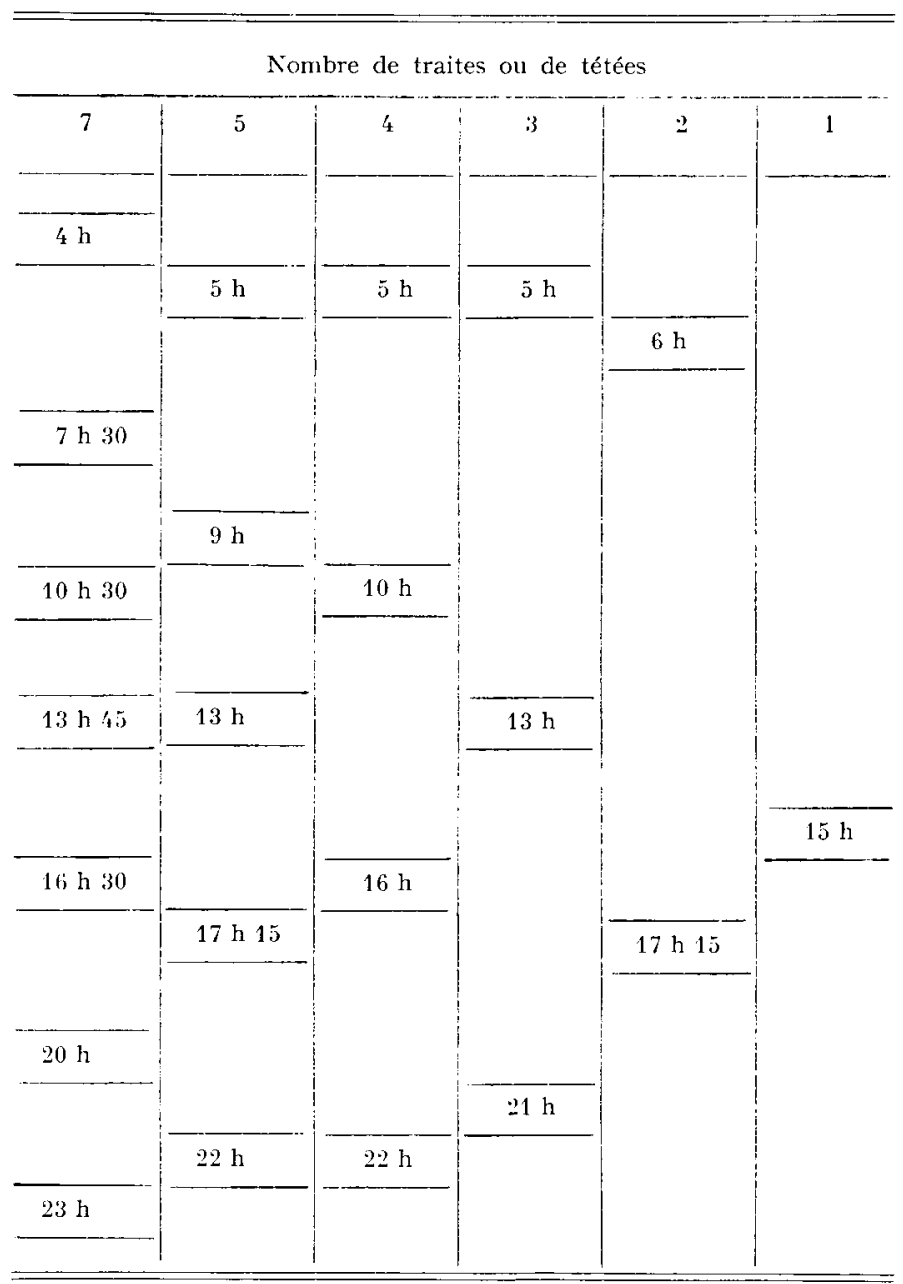

2. Contrôle de la quantité de lait trait.

La traite a lieu sur un "carroussel n à 32 places équipé d'une machine Alfa-Laval dont les caractéristiques sont les suivantes :

vide : $33 \mathrm{~cm} \mathrm{Hg}$

I 80 pulsations/minute

rapport succion-massage : 50 p. 100 . 
Les gobelets pesant environ $240 \mathrm{~g}$ sont toujours posés immédiatement sans massage ni préparation préalable de la mamelle. La finition de la traite est assurée par un égouttage à la machine puis 2 minutes environ après la dépose de la griffe par un égouttage manuel ou repasse. de $5 \mathrm{~g}$.

La production laitière de chaque brebis est pesée à chaque traite avec une précision voisine

\section{C. - Durée des périodes expérimentales et répartition des animaux dans les différents groupes}

La composition des groupes est présentée au tableau 2.

TABLEAU 2

Nombre de brebis dans chaque groupe et répartition selon le numéro de lactation

Nombre de brebis au cours de la première période

\begin{tabular}{|c|c|c|c|c|c|c|c|c|c|c|c|c|c|}
\hline & \multicolumn{6}{|c|}{ Groupes tétés (105) } & \multicolumn{7}{|c|}{ Groupes traits (93) } \\
\hline & A & $\mathrm{B}$ & $\mathrm{C}$ & $\mathrm{D}$ & $\mathrm{E}$ & $\mathrm{F}$ & $A^{\prime}$ & $\mathrm{B}^{\prime}$ & $C^{\prime}$ & $D^{\prime}$ & $E^{\prime}$ & ! & $F^{\prime}$ \\
\hline 2 lactation & 13 & 12 & 12 & 11 & 13 & 11 & 11 & 10 & 10 & 11 & $1 x^{\prime}$ & & 9 \\
\hline 3 e lactation & 5 & 6 & 5 & 7 & 5 & 5 & it & 5 & 5 & 5 & $\because$ & & 7 \\
\hline TOTAL & 18 & 18 & 17 & 18 & 18 & 16 & 15 & 15 & 15 & 16 & 16 & : & 16 \\
\hline
\end{tabular}

Nombre de brebis sur l'ensemble du carré latin (86)

\begin{tabular}{|c|c|c|c|c|c|c|c|c|c|c|c|c|c|}
\hline & \multicolumn{6}{|c|}{ Groupes tétés $(96)$} & \multicolumn{7}{|c|}{ Groupes traits } \\
\hline & A & $\mathrm{B}$ & $\mathrm{C}$ & D) & $\mathrm{E}$ & li & $A^{\prime}$ & $\mathrm{B}^{\prime}$ & $\mathrm{C}^{\prime}$ & | & $\mathrm{D}^{\prime}$ & $E^{\prime}$ & $\mathrm{I}^{\prime \prime}$ \\
\hline 2 lactation & 11 & 11 & 11 & 9 & 11 & 11 & 11 & 10 & 9 & & 10 & 13 & 5 \\
\hline $3^{e}$ lactation & 5 & 5 & 5 & 7 & 5 & 5 & $\ell^{\prime}$ & 5 & 5 & & $j$ & 2 & 7 \\
\hline TOTAL & 16 & 16 & 16 & 16 & 16 & 16 & 15 & 15 & 14 & ! & 15 & 15 & 13 \\
\hline
\end{tabular}

\section{Essai tétée.}

96 brebis sont divisées en 6 groupes égaux équilibrés en fonction de leur période d'agnelage.

Chaque période dure io jours à l'exception de la première d'entre elle qui est exceptionnellement allongée à 55 jours.

Cette première période constitue d'ailleurs à elle seule une comparaison simple dont les résultats ne peuvent être biaisés par les éventuels effets résiduels d'un traitement précédent.

On remarquera que dans un tel cas il a été possible de considérer 9 animaux supplémentaires (soit ro5 en tout) qui ont dû être éliminés par la suite pour des causes d'accident ou de maladie.

\section{Essai traite.}

Le plan expérimental permettant d'étudier l'influence de la fréquence des traites est sensiblement identique. La seule différence porte sur le nombre de brebis puisque 86 d'entre elles sont incorporées dans l'analyse du carré latin et 93 pour la comparaison des I 5 premiers jours. 


\section{III. - RÉSULTATS}

\section{A. - Évolution du lait trait et du lait tété au fur et à mesure de la succession des différents traitements}

Quelle que soit la succession des 6 traitements il apparait à la figure 2 que le lait recueilli par l'agneau et celui obtenu à la machine, puis à la repasse manuelle, évoluent selon 2 courbes très semblables et généralement superposables.
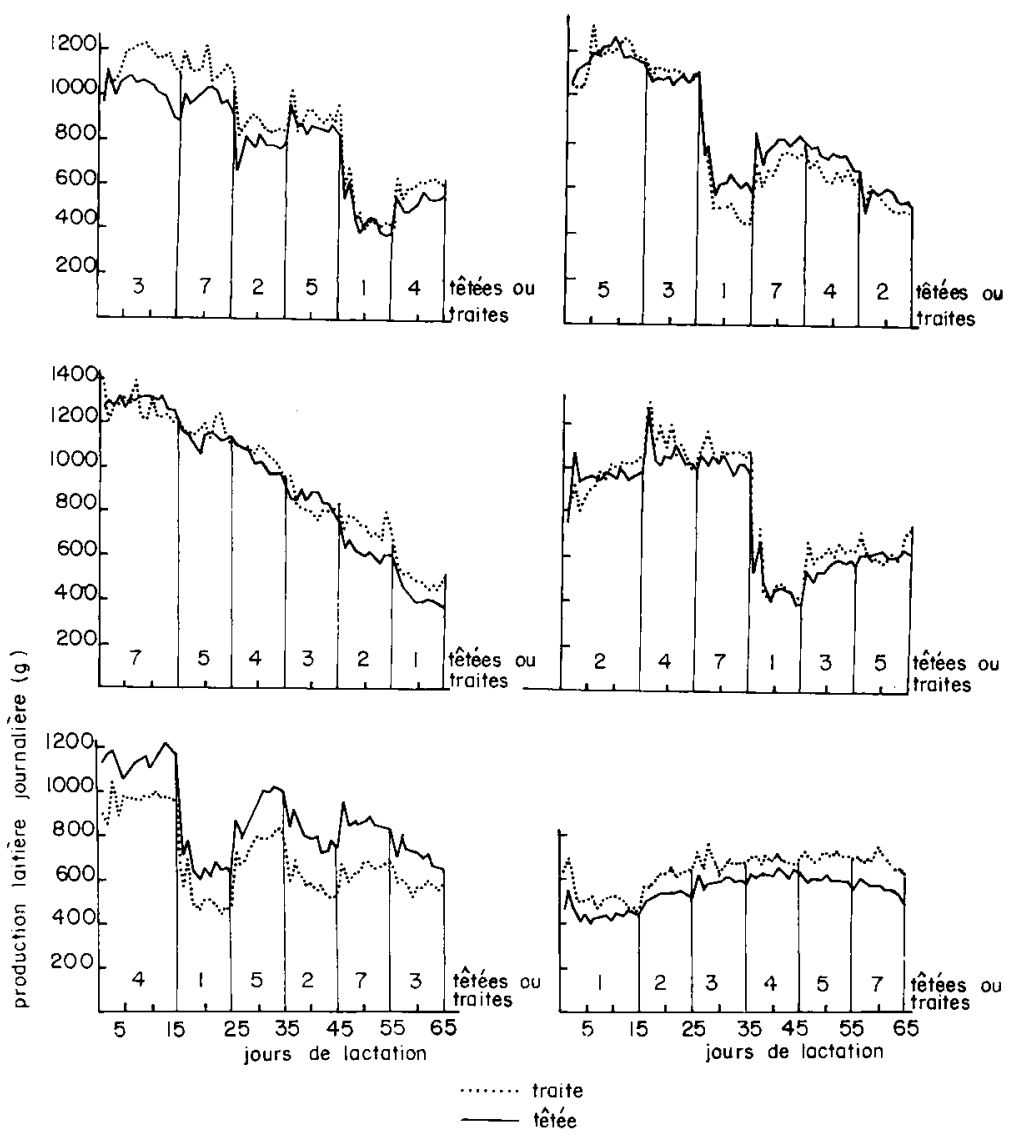

FIG. 2. - Effets de la succession des différentes fréquences de traites et de tétées sur la production laitière des brebis

On remarquera toutefois, l'effet dépressif très important provoqué par un nombre insuffisant de traite ou de tétée et ceci justifie l'utilisation d'un carré latin équilibré qui ne répète jamais les mêmes successions (fig. I). 


\section{B. - Analyse statistique de l'infuence des différentes fréquence's de traite et de tétéc}

I. Comparaison portant uniquement sur la première période expérimentale de 15 jours.

Afin de diminuer au maximum les erreurs pouvant résulter de certains phénomènes d'adaptation, nous avons préféré apprécier l'efficacité de chaque traitement en faisant, pour chaque brebis, la moyenne des productions laitières obtenues au cours des 8 derniers jours de cette première période expérimentale.

Les résultats sont présentés au tableau 3 et l'analyse de variance à une voie de classification indique que l'accroissement de la production laitière avec le nombre de traites ou de tétées est hautement significatif.

Le test de Newman et Keuls, que nous avons schématisé en incorporant dans une même accolade les fréquences qui ne diffèrent pas entre elles, permet d'apporter à ce sujet les renseignements supplémentaires sur la comparaison de celles-ci prises 2 à 2 .

\section{TABLEAU 3}

Influence du nombre de traites et de tétées sur la production laitière des brebis

(calculs effectués à partir des 8 derniers jours de la première période du carré latin) et signification des différences entre chaque tréquence de traite et de tétée

(test de Newman et Keuls)

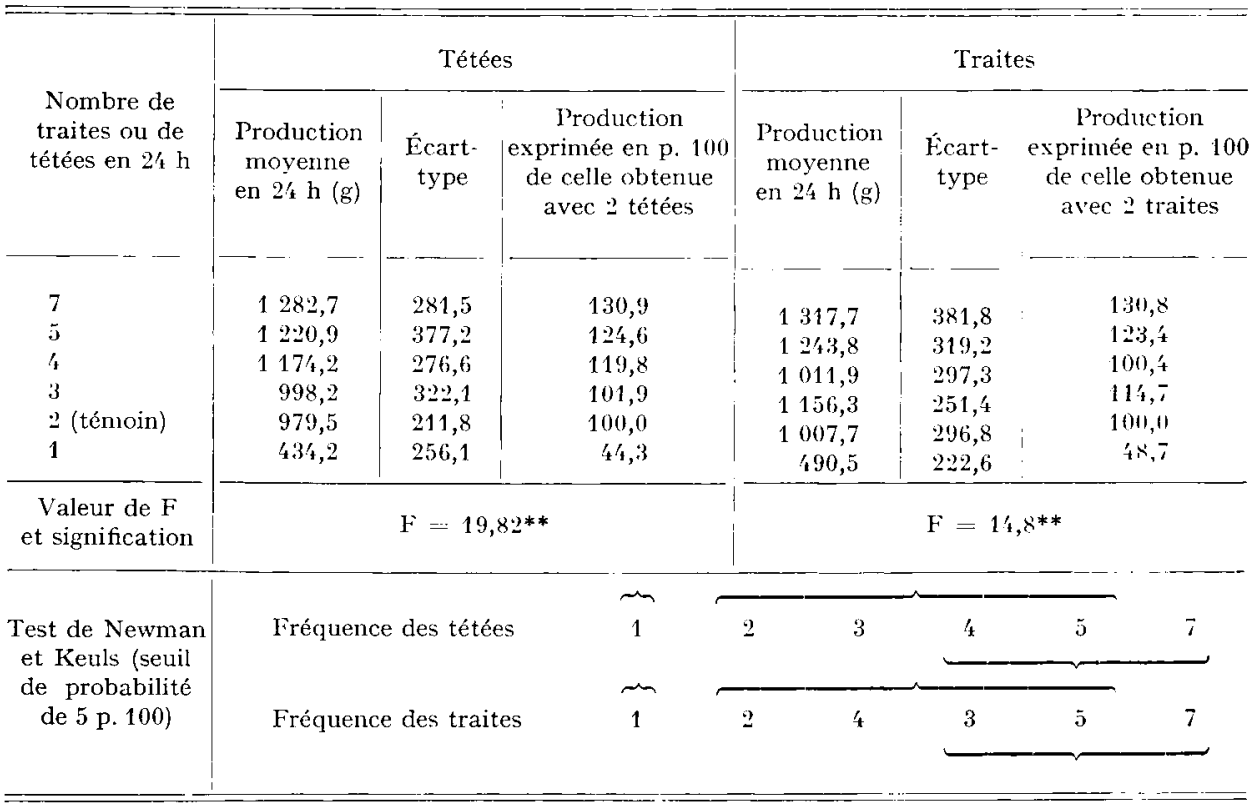

I1 apparaît surtout que par rapport au traitement 2, choisi arbitrairement comme témoin, la pratique d'une seule traite ou d'une seule tétée quotidienne entraîne une réduction importante de la production alors qu'inversement le " passage " 
à des fréquences supérieures conduit généralement à des gains relativement limités.

La superposition des courbes présentées à la partie supérieure de la figure 3 signifie que l'efficacité de l'agneau étranger n'est pas supérieure à celle de la machine lorsque le travail de celle-ci est complété par un égouttage manuel.

2. Comparaison portant sur l'ensemble du plan expérimental en carré latin.

Pour des raisons analogues à celles mentionnées précédemment nous n'avons retenu que les valeurs enregistrées au cours des 5 derniers jours de chaque période expérimentale.

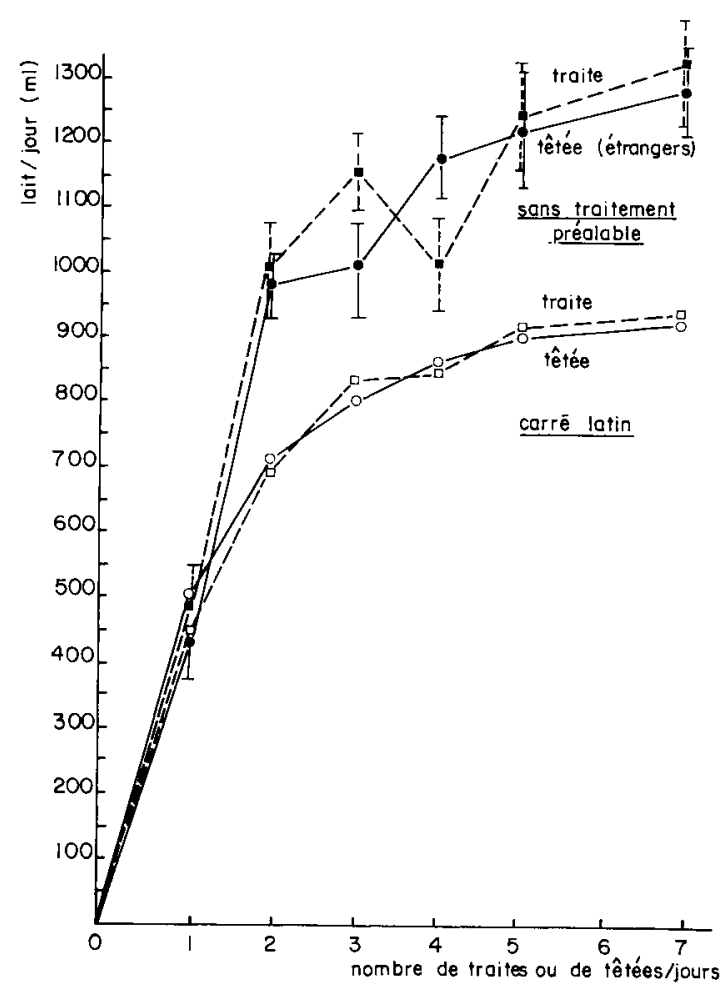

FIG. 3. - Influence de la fréquence des traites et des tétées sur la production laitière des brebis Préalpes du Sud

Le tableau 4 (auquel nous avons adjoint le détail de l'analyse de variance de chaque carré latin et la schématisation du test de Newman et Keuls) confirme la différence significative entre traitement déjà observée.

Il importe malgré tout de compléter ces résultats par 2 nouvelles remarques :

a) La sécrétion laitière est fortement freinée lorsque les brebis ne sont traites ou tétées qu'une seule fois par jour et tous les traitements qui sont appliqués par la suite se trouvent ainsi pénalisés par cet effet dépressif. A l'exception du traitement I, 
les valeurs relevées alors, sont donc biaisées par défaut et il est donc compréhensible que les tracés effectués dans ces conditions "plafonnent " plus rapidement (partie inférieure de la figure 3).

\section{TABLEAU 4}

Influence du nombre de traites et de tétées sur la production laitière des brebis (calculs effectués sur les 5 derniers jours de chaque période du carré latin)

Influence du nombre de tétées

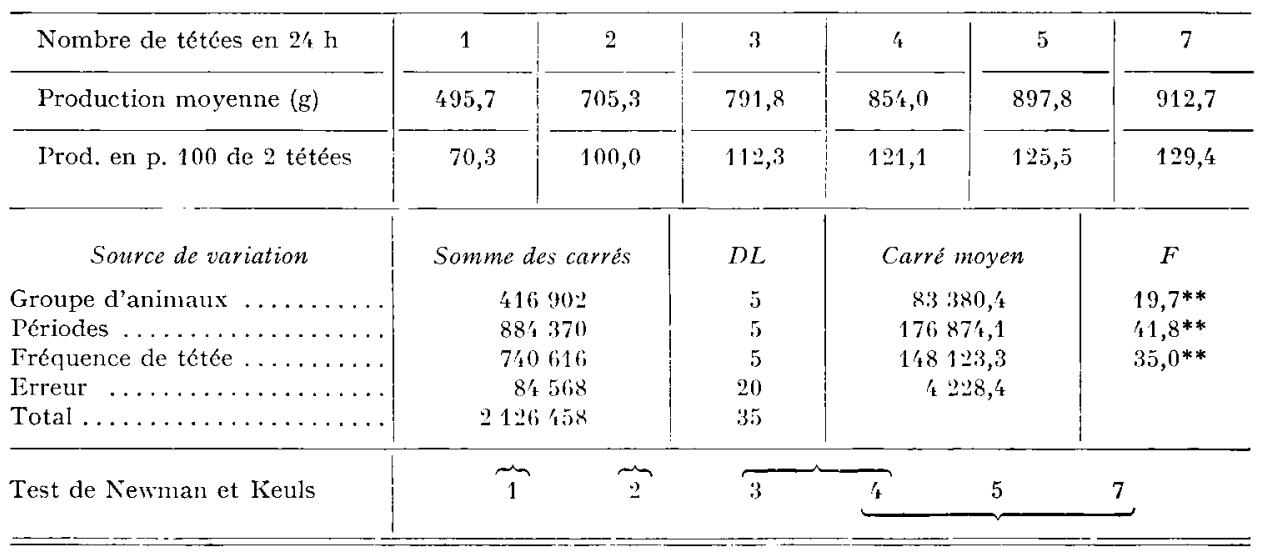

Influence du nombre de traites

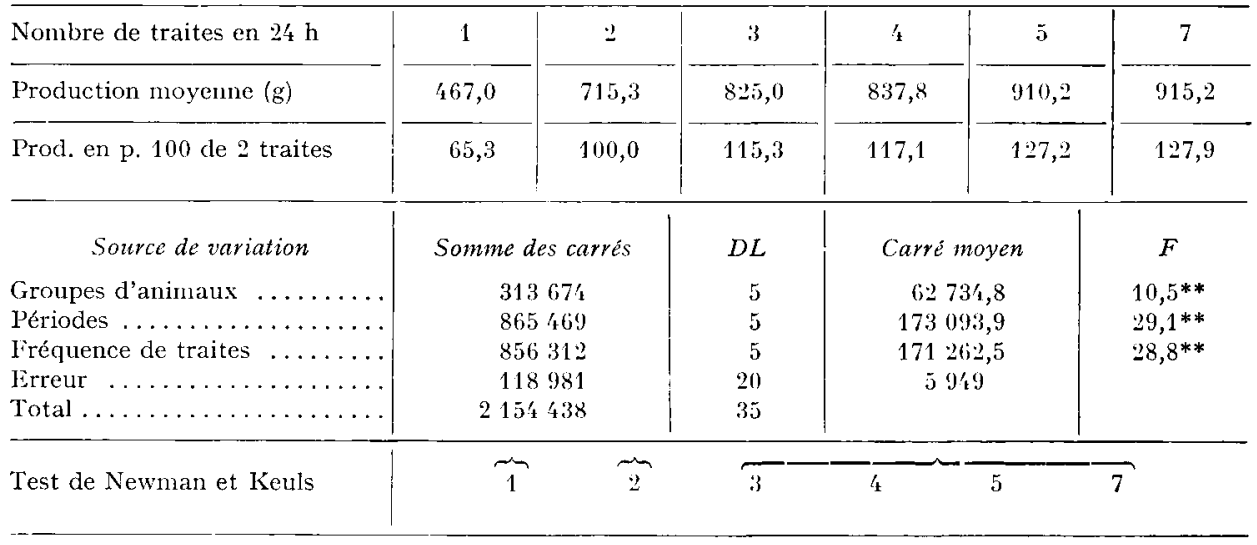

** : Signification au seuil 0,01 .

b) La similitude des courbes de production du lait trait et du lait tété ne signifie pas pour autant que la machine à elle seule est aussi efficace que l'agneau. En réalité, et comme l'indique la figure 4 le supplément de lait recueilli lorsque l'on passe de 2 à 7 traites par jour provient essentiellement des opérations d'égouttage manuel, puisqu'il apparaît que le volume de repasse augmente alors très sensiblement.

Annales de Zootechnie. - I974. 


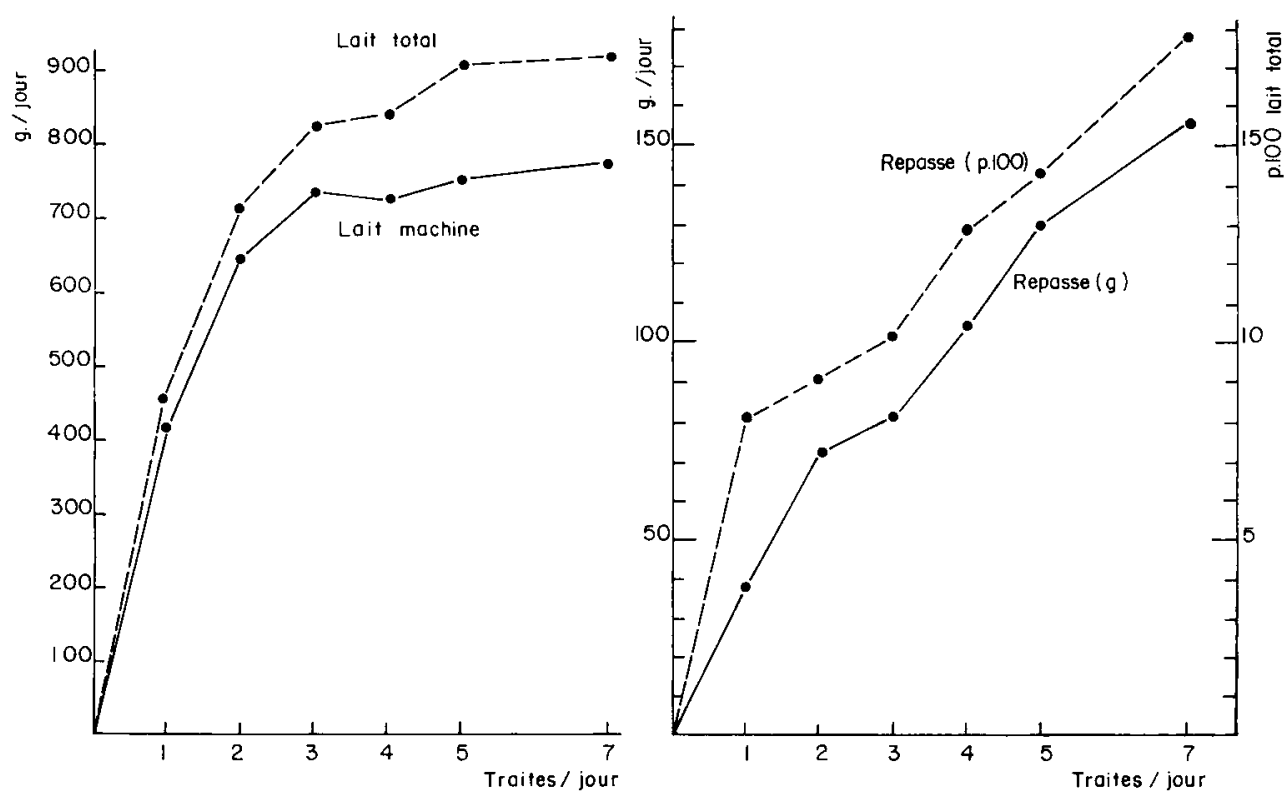

Iìg. 4. - Evolution du lait obtenu à la machine et à la repasse manuclle avec l'augmentation de la fréquence des traites

C. - Nombres d'agneaux nécessaires pour vider la mamelle

Le nombre d'agneaux nécessaires pour vider la mamelle est plus important lorsque ceux-ci sont encore jeunes et vigoureux et lorsque la fréquence quotidienne de tétée est faible (tabl. 5).

TABLEAU 5

Nombre d'agneaux nécessaires pour vider la mamelle; évolution en fonction de leur âge (période expérimentale) et de la fréquence quotidienne des tétées

\begin{tabular}{|c|c|c|c|c|c|c|c|}
\hline Nombre de tétées & 1 & 2 & 3 & 4 & 5 & 7 & Xoyenne \\
\hline $1^{\text {re }}$ période & 1,493 & 1,281 & 1,215 & $1,2^{\prime}+0$ & 1,120 & 1,044 & 1,2323 \\
\hline 2 période & 1,350 & 1,045 & 1,098 & 1,0114 & 1,017 & 1,017 & 1,091 \\
\hline 30 période & 1,225 & 1,060 & 1,030 & 1,017 & 1,012 & 1,005 & $1,0,58$ \\
\hline $4^{\circ}$ periode & 1,012 & $1,0 \div 27$ & $1,0 \pm 8$ & 1,050 & 1,008 & 1,008 & $1,0,22$ \\
\hline $5^{\mathrm{e}}$ période & 1,006 & 1,015 & 1,006 & 1,025 & 1,007 & 1,003 & 1,010 \\
\hline fo $^{\circ}$ période & 1,055 & 1,006 & $1,00_{4}^{\prime}$ & $1,00 / 4$ & 1,003 & 1,002 & 1,012 \\
\hline Moyenne & 1,191 & 1,072 & 1,063 & 1,058 & $1,0.27$ & 1,013 & 1,071 \\
\hline
\end{tabular}

C'est ainsi qu'au cours de la première période expérimentale, les brebis soumises à une seule tétée journalière ont dû allaiter environ un "agneau et demi ". Cette 
obligation est encore plus impérieuse au tout début de l'essai puisque les valeurs moyennes sont alors respectivement : $I, 75 ; 2,00$ et $I, 62$ pour les premier, deuxième et troisième jour.

\section{IV. - DISCUSSION ET CONCLUSION}

Les points les plus importants qui se dégagent de ce travail sont les suivants :

$\mathrm{I}^{\circ} \mathrm{A}$ fréquence égale de traite ou de tétée, le trayeur qui prend soin de pratiquer l'égouttage manuel après la dépose des gobelets peut obtenir une quantité de lait sensiblement comparable à celle recueillie par un agneau " étranger » dont l'efficacité physiologique au niveau extra-mammaire est probablement plus faible que celle de l'agneau " maternel ".

Toutefois, ceci ne signifie aucunement que la machine à traire et l'agneau maternel soient équipotents.

$2^{\circ}$ La différence de production constatée entre 7 traites et 2 tétées (I $317 \mathrm{~g}$ contre $979 \mathrm{~g}$ au cours de la première période, soit $26 \mathrm{p}$. 100 et $915 \mathrm{~g}$ contre $705 \mathrm{~g}$ sur l'ensemble du carré latin, soit 23 p. roo) permet d'expliquer, pour une grande part, la perte de production laitière d'environ $30 \mathrm{p}$. Ioo constatée au moment du sevrage.

Il convient d'ailleurs de souligner que cette différence est très certainement sous estimée car le lait tété aurait pu être bien supérieur si les contraintes expérimentales avaient moins perturbé les conditions naturelles d'allaitement.

$3^{0}$ Il se confirme que la race Préalpes $d u$ Sud supporte très difficilement la pratique d'une seule tétée ou d'une seule traite quotidienne. Lorsque ces interventions sont effectuées 2 fois par jour les quantités de lait récoltées sont, en effet, sensiblement accrues : respectivement $+55,7$ p. Ioo, $+5 \mathbf{I}, 3$ p. Ioo lorsque la comparaison porte sur la première période et $29,7 \mathrm{p}$. Ioo et $34,7 \mathrm{p}$. Ioo quand on prend en considération les valeurs enregistrées sur l'ensemble du carré latin. Nous retiendrons de préférence les chiffres issus des I5 premiers jours expérimentaux puisqu'ils ne sont pas biaisés par l'effet dépressif résiduel résultant d'une accumulation prolongée du lait dans la mamelle. La citerne de la Brebis Préalpes est en effet, peu volumineuse et cette caractéristique morphologique suffit peut-être à expliquer les divergences entre nos propres résultats et ceux précédemment obtenus à ce sujet par BAGDASAROV en I960 ( + r 8 p. xoo sur des animaux à toison fine) et par MoraG en I968 ( $+20 \mathrm{p}$. Ioo avec des races très diverses) et plus récemment par CASU et LABUSSIÈrE en I972 chez la Brebis Sarde ( +6 p. IOO).

$4^{\circ}$ Les gains de production que nous observons en passant de 2 à 3 traites (ou de 2 à 3 tétées) se situent entre I2 et $\mathrm{I} 5 \mathrm{p}$. Ioo. Ils sont donc supérieurs à ceux signalés par Semuan (I96I) sur des croisées Valache $\times$ Hampshire et inférieurs à ceux enregistrés par GAAL (I957) sur des brebis Tsigaia ou par Svec (Ig66) sur les produits d'un croisement Valache $\times$ Texel. Les remarques que nous avons formulées précédemment nous conduisent à prendre en considération la diversité des races et des plans expérimentaux pour interpréter ces différences. 


\section{SUMMARY}

\section{INFLUENCE OF THE FREQUENCY OF MILKINGS AND SUCKLINGS ON THE MILK YIELD OF PRÉALPES DU SUD EWES}

The eftects of the number of sucklings and mechanical milkings were studied by means of 2 latin squares, $6 \times 6$, each including :

- 6 treatments: $1,2,3,4,5$ and 7 milkings or sucklings per 24 hours. The time-table is given in table $\mathbf{I}$.

- 6 periods of ro days except for the first one lasting i 5 days and which, having not been affected by a previous treatment, constitute a very interesting experimental comparison.

- 6 groupes of Préalpes du Sud ewes, the composition of which is reported in table 2 (a total of 200 animals).

The results of this trial are given in figures 2 and 3 as well as in tables 3 and 4 . The examination of these data indicates that at the same frequency of milking and suckling, the efficiency of a "foreign "lamb does not exceed that of a milking machine when the working of the latter is completed by a manual intervention of the milker.

As compared to treatment 2, arbitrarily chosen as a control, one only milking or suckling per day results in a large reduction of the yield $\left(55.7\right.$ and $5^{\mathrm{I}} \cdot 3 \mathrm{P}$. Ico respectively when the comparison concerns the first period, and 29.7 and $34.7 \mathrm{p}$. Ioo when considering the values recorded on the whole latin square).

Conversely, the application of higher frequencies leads to relatively restricted gains (12 to I 5 p. Ioo between treatment $2 /$ day and $3 /$ day).

The difference of production obtained between 7 milkings and 2 sucklings accounts, to a great extent, for the decreasing milk production usually noticed at the moment of weaning.

\section{REMERCIEMENTS}

L'augmentation de la fréquence des traites et des tétées ayant imposé un grand nombre d'heures de travail nocturne nous tenons à remercier très vivement MM. BIDAURRETA, BoUSCARY, Brochet, Julien, Gavin, Girard, Gouget, lambion, Laxague, Teissoniere, Uhart qui ont bien voulu pendant plusieurs mois assurer la réalisation de ce travail expérimental particulièrement contraignant.

Cette publication leur est dédiée.

\section{RÉFÉRENCES BIBLIOGRAPHIQUES}

BaGdasarov G., I960. The milk production of fine wooled and Semi fine wooled ewes (en russe) Ovcevodstvo 6, 22-24 (cité par Boy azoglu J., Ann. Zootech., r963, 12, 237-296).

Casu S., Labussière J., ig72. Premiers résultats concernant la suppression d'une ou plusieurs traites par semaine chez la Brebis Sarde. Ann. Zootech., 21, 223-232.

GaAL M., I957. Ergebmisse des dreimaligen Melkens von Zigaja-Schafen in gross-betrieb (en Hongrois). Allattenyesztes, 6 (I), 6r-64

Grigorov H., Shalichev Y., I962. The effect of the Number of Milkings and of the différent intervals between them on the butter and protein content of the sheep milk obtained. XVI th Int. Dairy Congress Section I, 1, 258-264.

Labussière J., Combaud J. F., Petreguin P., i974. Effets de la suppression de la traite du dimanche soir chez la brebis Préalpes. Ann. Zootech., 23, $435-444$.

Labussière J., Petrequin P., ig69. Relations entre l'aptitude à la traite des brebis et la perte de production laitière constatée au moment du sevrage. Ann. Zootech., 18, 5-15. 
McCance I., 1959. The determination of milk yield in the merino ewe. Austr. J. Agric. Res. 10, $839-853$.

Morag M., 1966. A reduction in milk yield associated with certain half-udder milking techniques. $J$. Dairy Res., 33, 223-224.

MoraG M., I968. The effect of varying the daily milking frequency on the milk yield of the ewe and evidence on the nature of the inhibition of milk ejection by half-udder milking. Ann. Zootech., 17, $35 \mathrm{I}-369$.

Morag M., r969. The relation between the passage of time and the secretion of milk in the ewe. A ust. J. A gric., Rés., 20, 94 I-95I.

Munro J., Inkson R. H., 1957. The effects of different suckling frequencies on the quantity of milk consumed by young lambs. J. A gric. Sci., 49, r69-170.

RiCORdead G., Boccard R., Denamur r 960 . Mesure de la production laitière des brebis pendant la période d'allaitement. Ann. Zootech., 9, 97-1 20.

Semjan S., I96r. The problem of milking frequency of ewe. Pol nohospodarstvo Bratislava, 8 (2), 13I-I $3^{8}$. In Dairy Sci. Abst., 23, 3 I4.

SEMJAN S., rg6r. Influence of the milking interval on the milk yield of ewes. Sborn Vysokej skoly Pol' nohosp. Prevadzkovo Ekon Fak, 5, 229-236. (in Dairy Sci. Abst, 26, 367).

Semjan S., I962. Residual milk of sheep. XVIth Int. Dairy Congress. Section 1, 1, 17-24.

Svec J., 1966. A contribution to the number of milking of coarse wool sheep (cn tchèque). Vedecke Prace vyskumnebo ustavu ovciarskeho V. Trencine, 3, $23 \mathrm{I}-245$. 